\title{
Acute coronary occlusion secondary to radiofrequency catheter ablation of a left lateral accessory pathway
}

\author{
P. Chatelain, M. Zimmermann, R. Weber, C. Campanini and R. Adamec \\ Cardiology Center, University Hospital, Geneva, Switzerland
}

\begin{abstract}
KEY WORDS: Accessory pathway, radiofrequency ablation, complication, coronary occlusion.
A case of asymptomatic acute coronary occlusion secondary to radiofrequency catheter ablation of a left lateral accessory pathway is reported. Due to post-procedural ST modifications of the surface ECG, a coronary angiography was performed which disclosed total occlusion of the first marginal branch of the left circumflex coronary artery. Acute myocardial infarction was confirmed by moderate cardiac enzyme release, abnormal myocardial perfusion scan and mild lateral hypokinesia at echocardiography. This rare but potentially harmful complication of interventional electrophysiology should be kept in mind and coronary angiography performed immediately when coronary occlusion related to radiofrequency application is suspected.
\end{abstract}

\section{Introduction}

Radiofrequency ablation of accessory pathways is a recognised definitive therapy of tachyarrhythmias occurring within the Wolff-Parkinson-White syndrome ${ }^{[1.2]}$. Recently, the Multicentre European Radiofrequency Survey (MERFS) has reported complications in 4398 of these procedures ${ }^{[3]}$. However, acute coronary occlusion secondary to catheter ablation was not among them. We report a case of acute circumflex coronary artery occlusion secondary to radiofrequency catheter ablation of a left lateral accessory pathway.

\section{Case report}

A 45-year-old woman was referred to our institution for recurrent symptomatic supraventricular reentrant tachycardia secondary to a left lateral accessory pathway first documented in 1988. Initially, the patient was treated with various drugs including propafenone, flecainide and amiodarone without satisfactory clinical improvement. Because of significant alterations in thyroid function under amiodarone therapy, thermoablation of the accessory pathway was proposed.

The resting ECG showed a patent permanent WolfParkinson-White aspect with a delta wave in leads II, III, aVF, and from $V_{1}$ to $V_{6}$ (Fig. 1 upper panel). A new electrophysiological study confirmed the presence of a left lateral accessory pathway with a maximal anterograde conduction of 180 beats $\min ^{-1}$ and a maximal retrograde conduction of 290 beats . min $^{-1}$. Orthodromic reentrant supraventricular tachycardia was easily inducible as was atrial fibrillation.

It was decided to proceed with thermoablation. The ablation catheter (7F Webster, large tip, no thermistor)

Revision submitted 2 September 1994 and accepted 5 September 1994.

Correspondence: P. Chatelain, MD, Cardıology Center, University Hospital Geneva, 1211 Geneva 14, Switzerland.
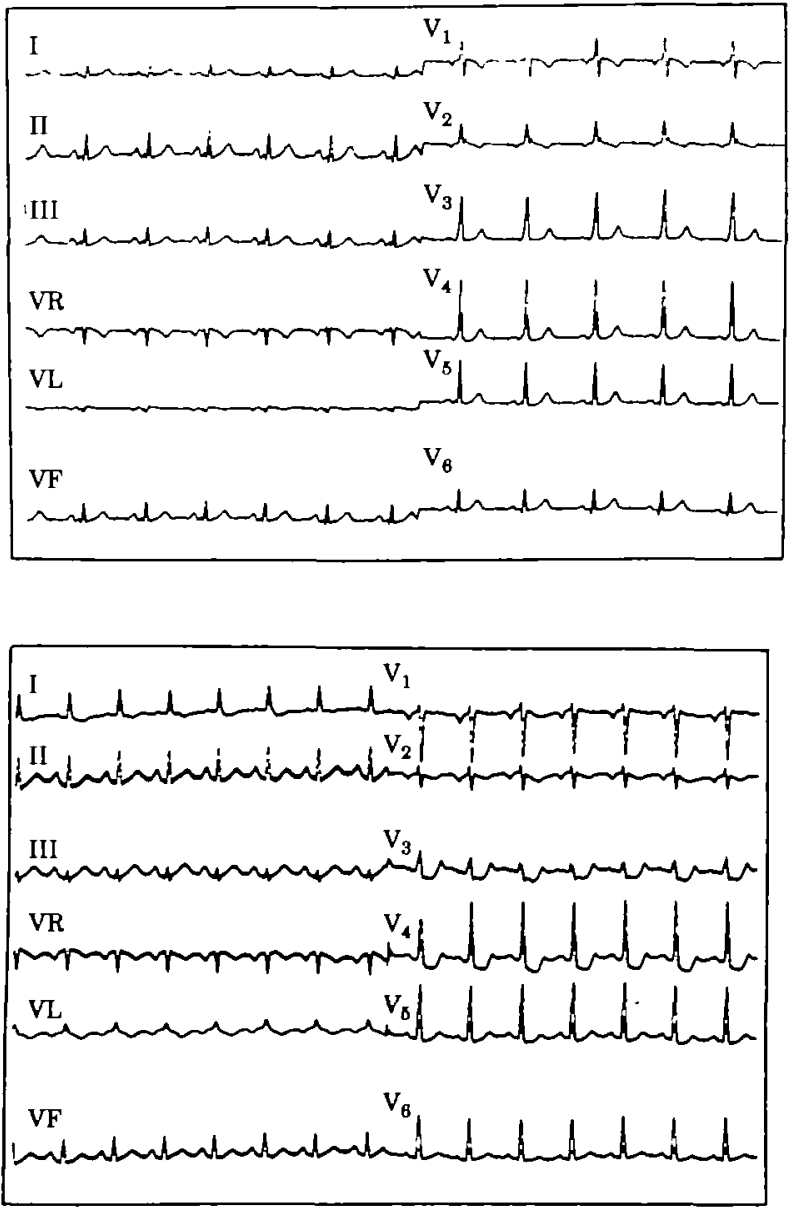

Figure I Upper panel: 12-lead electrocardiogram of the patient before the procedure showing sinus rhythm at 84 beats. $\mathrm{min}^{-1}$ and positive delta waves in leads $I I, I I I, a V F$, and from $V_{1}$ to $V_{6}$. Lower panel: 12-lead electrocardiogram after the ablation procedure showing disappearance of the delta wave and significant ST changes in leads $V_{2}$ to $V_{4}$. 

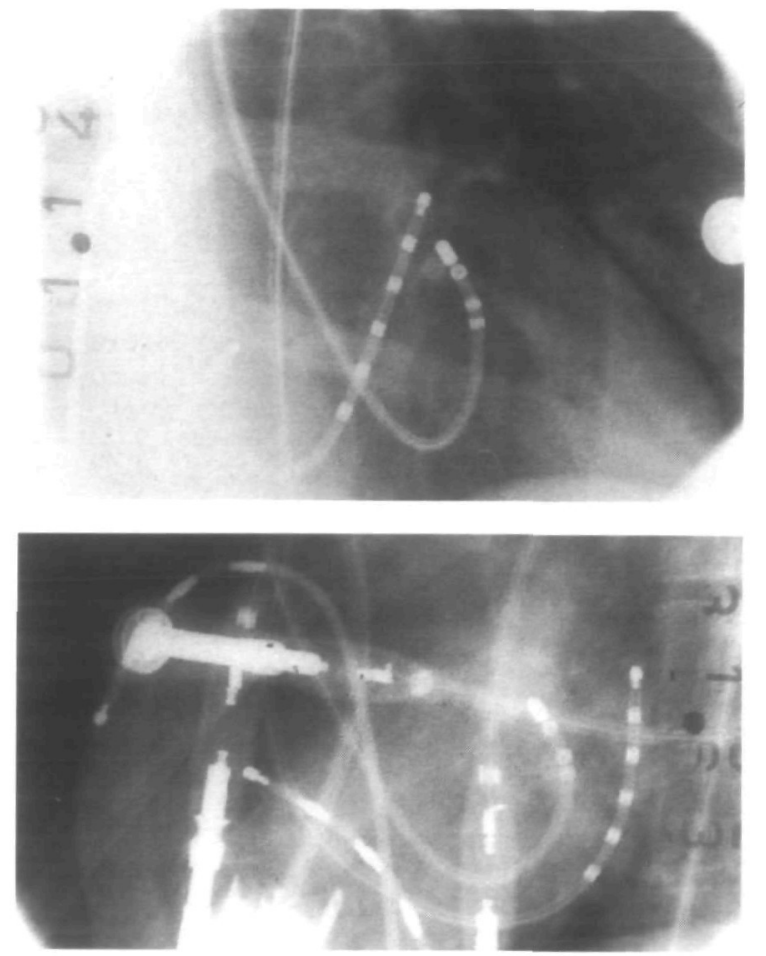

Figure 2 Right anterior oblique (top) and lateral (bottom) views of the thermoablation catheter in place in the ventricular side of the mitral annulus.

was inserted in the right femoral artery and a hexopolar recording catheter was inserted in the right jugular vein to record in the coronary sinus (Fig. 2). After easy localization of the left lateral accessory pathway, several shots were applied over a relatively localized area of the endocardium under the mitral annulus (retrograde transaortic approach). Each shot was of $30 \mathrm{~s}$ duration with a delivered energy varying between 28 and $43 \mathrm{~W}$. After six of the 17 shots, transient disappearance of the pre-excitation was noted. The catheter was slightly repositioned after each unsuccessful radiofrequency application. No impedance rise was observed. After the 17 th shot (final because it was successful) fast and permanent disappearance of the preexcitation was achieved. Following the procedure, a 12-lead ECG (Fig. 1 lower panel) showed ST changes in leads $V_{3}$ to $V_{5}$ attributed to a Chatterjee effect related to transient left bundle branch block observed during the procedure. The patient was asymptomatic. A control coronary angiogram was performed, which was initially considered normal, but disclosed, after review of the cinefilm, total occlusion of the first marginal branch of the circumflex coronary artery (Fig. 3). Non-Q wave myocardial infarction was confirmed by moderate increase of creatine kinase and SPECT imaging. Pericardial rub was absent as was pericardial effusion at echocardiography, which disclosed mild lateral hypokinesia. No other complication was observed and the patient was asymptomatic at discharge 6 days later.

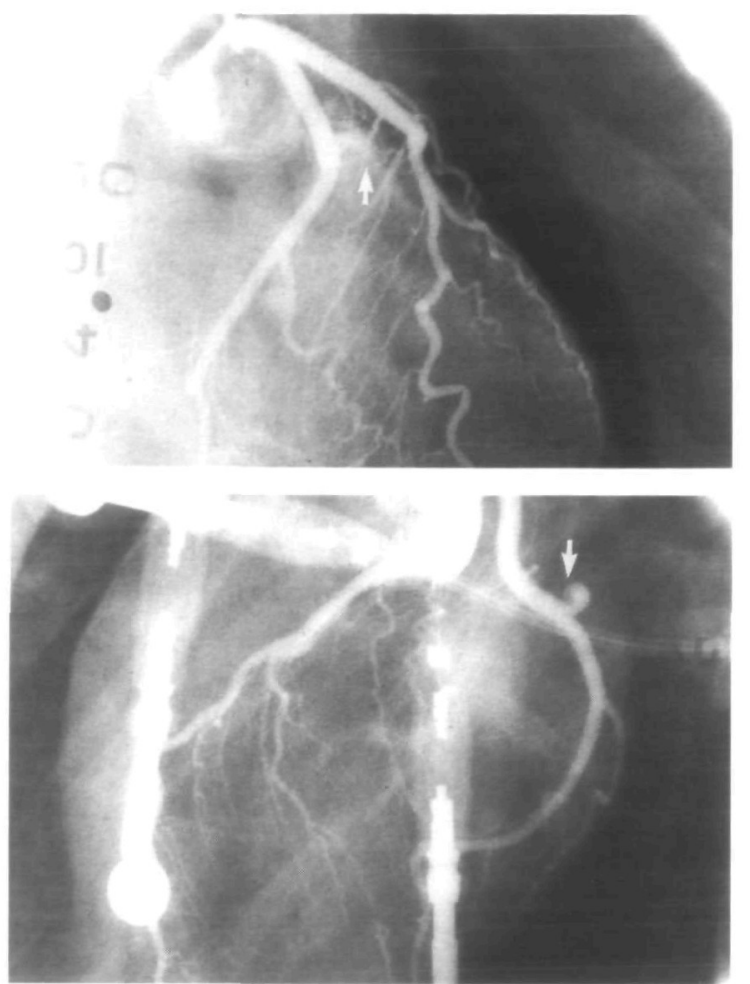

Figure 3 Biplane left coronary angiogram showing total occlusion of the first marginal branch of the circumflex coronary artery near its origin (arrows).

\section{Discussion}

In this case, acute coronary occlusion was thought to be secondary to application of radiof requency current during thermoablation of a left lateral accessory pathway. Comparison of the coronary angiogram with X-ray views of the ablation catheter in place disclosed anatomical correspondence between the latter and the marginal branch of the circumflex artery in which occlusion occurred. The mechanism responsible for the occlusion is not known and a recent study ${ }^{[4]}$ failed to demonstrate any short-term effect of radiofrequency current delivery on coronary artery anatomy as assessed angiographically. However, by analogy to surgical techniques, it is suspected that electrocoagulation occurred in the artery leading to thrombotic occlusion.

After review of the medical literature, it appears that acute coronary occlusion as a complication of radiofrequency ablation of accessory atrioventricular pathways is rare. Indeed, the recent MERFS survey ${ }^{[3]}$ and Scheinmann ${ }^{[S]}$ reported, respectively, a $4.4 \%$ and a $3 \cdot 8 \%$ incidence of complications in large series, but acute coronary occlusion was not among them. However, a case similar to ours (left free wall accessory pathway, left ventricular side current application, and left circumflex coronary artery occlusion) has been reported by Calkins et $a !^{[6]}$. In that particular case, acute occlusion was successfully treated by coronary angioplasty $30 \mathrm{~min}$ later. However, significant creatine kinase increase and 
mild left ventricular dysfunction occurred thereafter. In our case, because of off-line diagnosis of the complication and relatively small size of the coronary vessel in which occlusion occurred, there was no attempt to recanalize it.

A later publication of the group mentioned above ${ }^{[7]}$ has reported another case of an ischaemic complication after delivery of radiofrequency current in a similar situation. However, in that case, as in one reported by Lesh et al. ${ }^{[8]}$, a coronary angiogram performed immediately after was normal.

\section{References}

[1] Schlūter $M$, Geiger $M$, Siebels J, Duckeck W, Kuck K-H. Catheter ablation using radiofrequency current to cure symptomatic patients with tachyarrhythmias related to an accessory atroventricular pathway. Circulation 1991; 84. $1644-61$.

[2] Jackman WM, Wang X, Fnday KJ et al. Catheter ablation of accessory atrioventricular pathways (Wolff-Parkinson-White syndrome) by radiofrequency current. $\mathrm{N}$ Engl J Med 1991; 324 1605-11.
[3] Hindricks $G$ and the MERFS investigators. The Multicenter European Radiofrequency Survey (MERFS): complications of radiofrequency catheter ablation of arrhythmias. Eur Heart J 1993; 14: 1644-53.

[4] Solomon AJ, Tracy CM, Swartz JF, Reagan KM, Karasık PE, Fletcher RD. Effect on coronary artery anatomy of radiofrequency catheter ablation of atrial insertion sites of accessory pathways. J Am Coll Cardiol 1993; 21: 1440-4.

[5] Scheinmann MM. Radiofrequency catheter ablation for patients with supraventncular tachycardia. Learning Highlights of the American College of Cardiology 1992; Summer 1992: 8-14.

[6] Calkins H, Sousa J, El-Atassi et al. Diagnosis and cure of the Wolff-Parkinson-White syndrome or paroxysmal supraventricular tachycardias during a single electrophysiologic test N Engl J Med 1991; 324: 1612-8.

[7] Calkins H, Langberg J, Sousa J et al. Radiofrequency catheter ablation of accessory atrioventricular connections in 250 pattents. Circulation 1992; 85. 1337-46.

[8] Lesh MD, Van Hare GF, Schamp DJ et al. Curative percutaneous catheter ablation using radiofrequency energy for accessory pathways in all locations: results in 100 consecutive patients J Am Coll Cardıol 1992; 19: 1303-9. 